\title{
Fault Diagnosis of Power Transformer based on Probability-box Theory
}

\author{
Ding Jiaman ${ }^{1}$ \\ Yunnan Provincial Key Laboratory of Computer Technology \\ Application, Faculty of Information Engineering and Automa- \\ tion \\ Kunming University of Science and Technology \\ Kunming, China \\ e-mail: tjom2008@163.com
}

\author{
$\mathrm{Du} \mathrm{Yi}{ }^{2 *}$ \\ City College \\ Kunming University of Science and Technology \\ Kunming, China \\ e-mail: duyi215@hotmail.com
}

\author{
Wang Qingxin ${ }^{3}$ \\ Faculty of Information Engineering and Automation, \\ Kunming University of Science and Technology, \\ Kunming, China \\ e-mail: yunnan403@sina.com
}

\begin{abstract}
In practice, fault diagnosis of power transformer is often performed on the basis of limited data and many various uncertainties factors. Under this circumstance, there are practical difficulties in identifying unique distributions as input for fault diagnosis. In order to solve the problem and improve the diagnosis ability of power transformer by analyzing the dissolved gas, a new method based on probability boxes theory was proposed. Firstly, the raw percentages of dissolved gas were used as the information source to construct the tow pboxes about $\mathrm{H}_{2}$ and $\mathrm{C}_{2} \mathrm{H}_{6}$ gas content. Then, to take advantage of the complementation of the information source, the tow $p$ boxes about $\mathrm{H}_{2}$ and $\mathrm{C}_{2} \mathrm{H}_{6}$ gas content were fused. Finally, the SVM features database was established by extracting different types of cumulative uncertainty measures from p-boxes. The analysis result shows that the proposed method has high degree of diagnosis accuracy and is characterized by fast diagnosis and good real-time, demonstrating the model is practical and effective.
\end{abstract}

keywords- Fault diagnosis; Power transformer; Uncertainty; Probability box; Fusion

\section{INTRODUCTION}

Stable and sustainable performance of power transformer is the key to profitable generation and transmission of electric power. Failure of a large power transformer not only results in the damage of expensive equipment, but also can cause enormous casualties and economic losses. In recent years, domestic and foreign scholars had done many related works about the

\author{
Jia Lianyin ${ }^{4}$ \\ Yunnan Provincial Key Laboratory of Computer Technology \\ Application, Faculty of Information Engineering and Automa- \\ tion \\ Kunming University of Science and Technology, \\ Kunming, China \\ e-mail: yunnan403@ sina.com

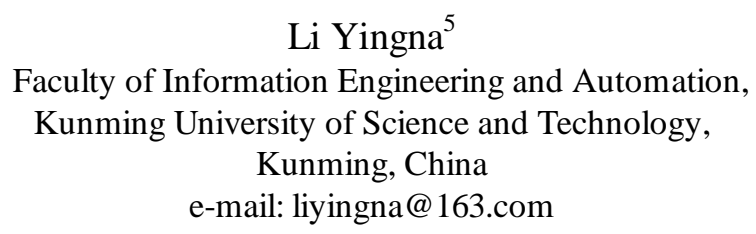

power transformer fault diagnosis by analyzing the dissolved gas in oil as a characteristic quantity [1-3]. The most common fault diagnosis method of power transformer is based on the Dissolved Gas-in-oil Analysis (DGA) of transformer oil. It is a sensitive and reliable technique for the detection of incipient fault condition within oil-immersed transformers. There are a number of methods developed for analyzing these gases and interpreting their significance such as Key Gas, Roger gas ratio, Doernenburg, IEC gas ratio and Duval Triangle. Although DGA has widely been used in the industry, this conventional method fails to diagnosis in some cases. This normally happens for those transformers which have more than one type of fault at the same time. To overcome this limitation, an expert system based on DGA for diagnosis of power transformer condition is proposed [4], in which combines three different DGA methods in one diagnosis scheme in order to overcome the limitation of each method stand alone and investigates the accuracy and consistency of three methods in interpreting the transformer condition by applying fuzzy logic technique in addition to a new final combined fuzzy system.

Above-mentioned research has made some achievements by analyzing independent effect of each uncertainty factor. But it is a key to diagnose fault of power transformer by analyzing the links between the various uncertainties factors.

As we all know, the uncertainties in nature may be divided into two kinds [5]. The one is the objective uncertainty from the time spatial variation, the material non-uniformity, etc., which is 
unable to change. The other is the subjective uncertainty from the cognition lacks, the metering equipment difference, etc., which is able to change. The thought of probability boxes (pboxes for short) originated in the expressed "cognitive uncertainty" with "interval". The probability box (p-box) theory can deal with above two kinds of uncertainties. Along with the uncertainty project' advancement of American Sandia Country Laboratory in 2004, the project promotions in finance risk analysis, biogeography and mechanical reliability and so on are getting more and more widespread [6]. The application of p-box theory successively involves the fault system failure probability assessment [7], dynamic responses of the vibration system uncertainty evaluation [8], the expression of uncertainty about climate change [9], Many parameters of seawall risk modeling and reliability assessment $[10,11]$, the lack of experimental data under the condition of automobile gearbox reliability design [12], rocket edge shell structure finite element modeling and parameter optimization [13]

Currently SVM has been widely used in face recognition, fault classification, nonlinear system modeling and identification and other fields [14].

\section{BASIC CONCEPTS}

\section{A. Probability box(p-box)}

Suppose $\bar{F}(x)$ and $\underline{F}(x)$ are increasing function of real numbers, which ranges are in $[0,1]$. For all $\mathrm{x} \in \mathfrak{R}$, there exists the relationship $\underline{F}(x) \leq \bar{F}(x)$. The interval $[\underline{F}(x), \bar{F}(x)]$ presents the upper and lower bounds of increasing function $\mathrm{F}(\mathrm{x})$ and satisfies the condition $\underline{F}(x) \leq F(x) \leq \bar{F}(x)$. The range $[\underline{F}(x), \bar{F}(x)]$ is called as a pair of function with an uncertainty distribution, which is called as a probability box.

For a random variable $\mathrm{X}$, assuming the probability distribution of $\mathrm{X}$ is unknown, $\underline{F}(x)$ is defined as the lower Cumulative distribution function (CDF) which presents the probability of $\mathrm{X} \leq \mathrm{x}$. Similarly, $\bar{F}(x)$ is defined as the upper CDF. The upper and lower bounds of the probability distribution function can be calculated by the probability measure $\underline{P}(x)$ :

$$
\begin{gathered}
\bar{F}_{X}(X)=1-\underline{P}(X>x) \\
\underline{F}_{X}(X)=\underline{P}(X \leq x)
\end{gathered}
$$

As shown in Fig .1, the left boundary $\bar{F}(x)$ is the upper boundary of the probability distribution, corresponding in the lower boundary of quantile $\mathrm{x}$. The right boundary $F(x)$ is the lower boundary of the probability distribution, corresponding in the upper boundary of quantile $\mathrm{x}$.

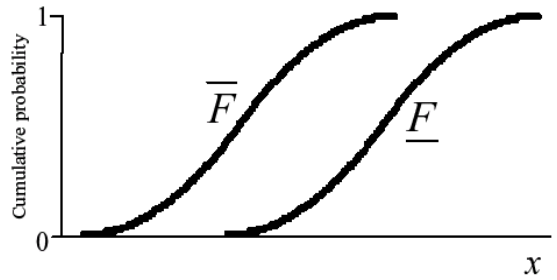

Figure1. An example of p-box

\section{B. Dempster Shafer Structure(DSS)}

A finite DSS on the real number field $\mathfrak{R}$ can be presented by its basic probability assignment function, which is a mapping relation:

$$
m: 2^{\Re} \rightarrow[0,1]
$$

Where:

$m(\Phi)=0$

$m\left(a_{i}\right)=p_{i}\left(a_{i} \subseteq \mathfrak{R}, i=1,2 \ldots n\right) \quad m(D)=0$

$p_{i}>0$

$\sum_{i=1}^{n} p_{i}=1$

The plausibility function (PIs):

It's a sum of all the corresponding mass of a DSS which has intersection with the subset $b \subseteq \Re$, which can be presented by the following function:

$$
P l s(b)=\sum_{\substack{a \\ a \cap b \neq \varnothing}} m(a)=\sum_{\substack{i \\ a_{i} \cap b \neq \varnothing}} m\left(a_{i}\right)
$$

The belief function (Bel):

It's a sum of all the corresponding mass of a DSS which is the subset of interval $b \subseteq \mathfrak{R}$, which can be presented by the following function:

$$
\operatorname{Bel}(b)=\sum_{\substack{a \\ a \subseteq b}} m(a)=\sum_{\substack{i \\ a_{i} \subseteq b}} m\left(a_{i}\right)
$$

A DSS is composed by the sets which including an interval and its corresponding mass. The form is as follows $\left\{\left(\left[\mathrm{x}_{1}, \mathrm{y}_{1}\right]\right.\right.$, $\left.\left.\mathrm{m}_{1}\right),\left(\left[\mathrm{x}_{2}, \mathrm{y}_{2}\right], \mathrm{m}_{2}\right), \ldots,\left(\left[\mathrm{x}_{\mathrm{n}}, \mathrm{y}_{\mathrm{n}}\right], \mathrm{m}_{\mathrm{n}}\right)\right\}$ and satisfying the following conditions:

$$
\begin{aligned}
& x_{i}<y_{i}, \\
& \sum m_{i}=1, \\
& y_{i} \neq y_{j} .
\end{aligned}
$$

\section{The Relationship of P-box and DSS}

The lower bound of a p-box can be achieved by the sum of all the corresponding lower bounds of a DSS and the upper bound of a p-box can be achieved by the sum of all upper bounds of a DSS. If a DSS can be presented by $\left\{\left(\left[\mathrm{x}_{1}, \mathrm{y}_{1}\right], \mathrm{m}_{1}\right)\right.$, $\left.\left(\left[\mathrm{x}_{2}, \mathrm{y}_{2}\right], \mathrm{m}_{2}\right), \ldots,\left(\left[\mathrm{x}_{\mathrm{n}}, \mathrm{y}_{\mathrm{n}}\right], \mathrm{m}_{\mathrm{n}}\right)\right\}$, the lower bound (Bel) function and the upper bound function of the corresponding p-box transformed from the DSS can be expressed by: 


$$
\begin{aligned}
& \underline{F}(z)=\operatorname{Bel}(z)=\sum_{y_{i}<z} m_{i} \\
& \bar{F}(z)=\operatorname{Pls}(z)=\sum_{x_{i}<\mathrm{z}} m_{i}
\end{aligned}
$$

The transformation process from a DSS to a p-box is not all information reserving. Some information in details will be lost. The p-box can also be transformed into a DSS by standard discretization.

\section{FAUlt Signal MOdELING BASED ON P-BOX THEORY}

\section{A. Construction of the p-boxes}

Four patterns involve normal state, discharge faults, overheated faults and grounding faults. The $\mathrm{H}_{2}$ and $\mathrm{C}_{2} \mathrm{H}_{6}$ two sorts' gas signals are picked up.

The steps in getting the p-boxes are shown as following:

1) Divide the data into several groups according to the sampling frequency.

2) Work out every group's mean and variance.

3) Get the DSS as given format.

4) Discretizing of the DSS.

5) Construct the p-boxes

The results of the p-boxes of $\mathrm{H}_{2}$ gas are showed on Fig .2.

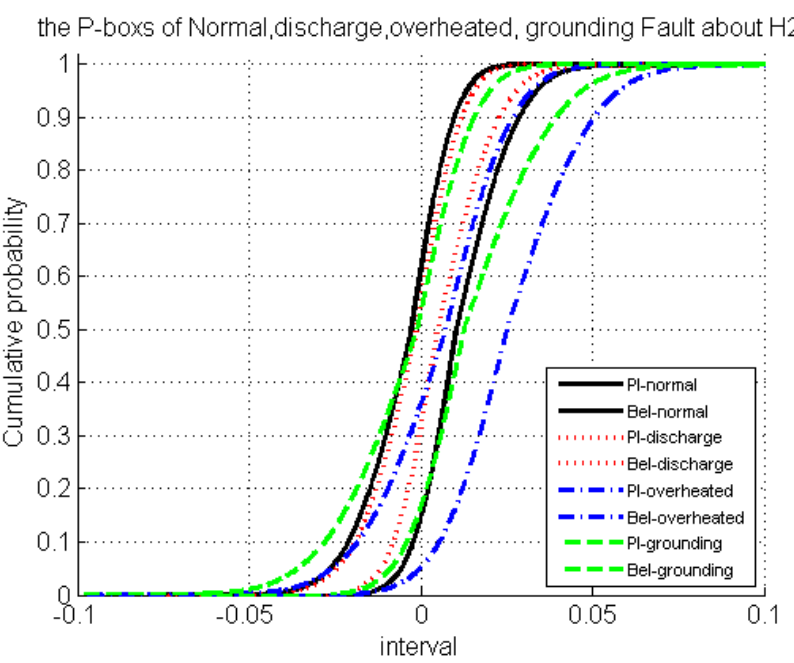

Figure2. The p-boxes of $\mathrm{H} 2$ gas

According to the above five steps, we get the results of the p-boxes of $\mathrm{C}_{2} \mathrm{H}_{6}$ gas are showed on Fig.3.

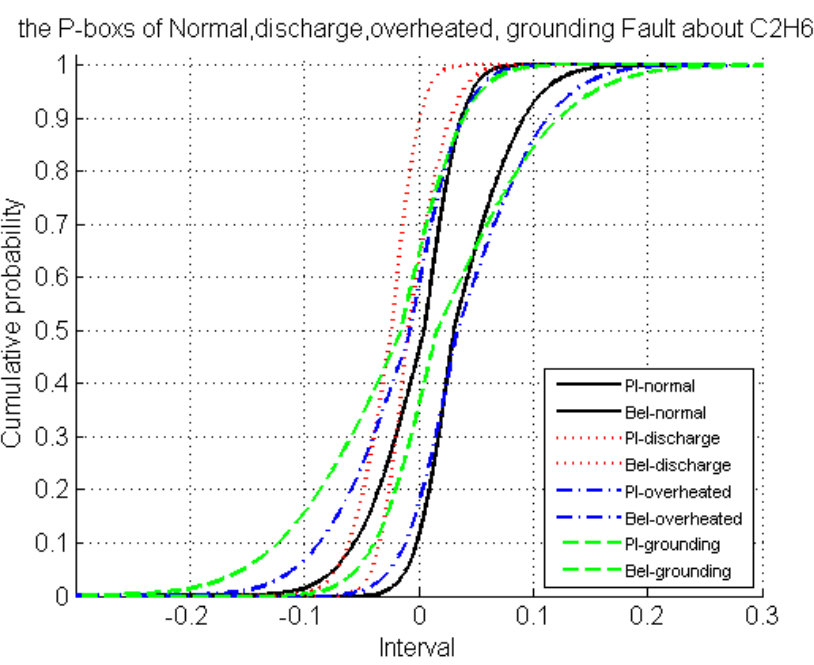

Figure3. The p-boxes of $\mathrm{C}_{2} \mathrm{H}_{6}$ gas
It is apparent from Fig .2 that the results are different from the Fig .3. The p-boxes are wider than the former. As we all know the fewer the data, the more uncertainty is the p-box.

\section{B. Fusion from the p-boxes}

Taking into account the gas content correlation and complementation between $\mathrm{H}_{2}$ and $\mathrm{C}_{2} \mathrm{H}_{6}$, It is necessary to fuse the information from $\mathrm{H}_{2}$ and $\mathrm{C}_{2} \mathrm{H}_{6}$ with the Demspter combination rules. The fusion results are showed on Fig 4 .

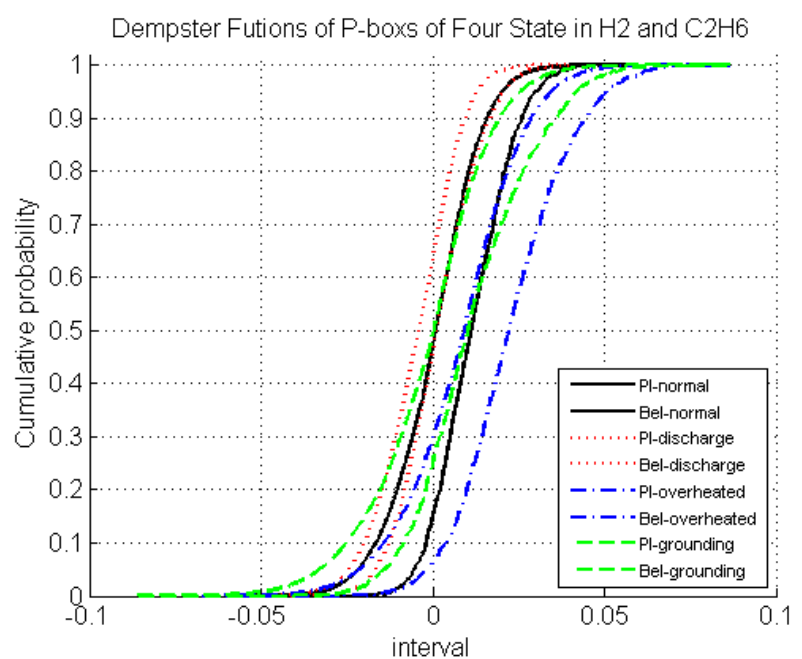

Figure4. The fused p-boxes of $\mathrm{H}_{2}$ and $\mathrm{C}_{2} \mathrm{H}_{6}$

It is apparent from Fig .4 that the compactness of the pboxes graphics has been improved, which means that we can more easily distinguish the four patterns through the Dempster fusion p-boxes.

\section{SVM DIAGNOSING BASED ON P-BOXES}

Support Vector Machine (SVM) is first put forward by Cortes and Vapink. Despite its short history, it has shown great advantages in the realm of machine learning. SVM excels in addressing high-dimension problem and solving the problem of small sample. In practice, fault diagnosis of power transformer is often performed on the basis of limited data, and SVM algorithm have the advantage in response to the small sample data using support vector to complete linear or non-linear programming problems. So in this paper the SVM method is used here to solve the problem about the p-boxes fault diagnosis of power transformer. ing:

The process of SVM pattern recognition is shown as follow-

1) Establish the training database and testing database. Training data and testing data should be constructed as the same time and randomly selected.

2) Data normalization. The feature input should be normalized to a lesser extent.

3) Select the kernel function and the parameters of SVM.

4) Train the SVM by using the training database to get the trained Pattern.

5) Enter the testing data, using the trained Pattern to obtain the classification results (classification accuracy).

The core of the function is the establishment of SVM features database from p-boxes.

\section{A. Establishment of features database}

The cumulative uncertainty measures methods are to get

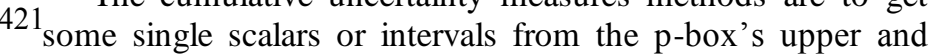


lower bounds. In this paper, the five features which are extracted from the five different types of cumulative uncertainty measures of p-boxes are used as the SVM pattern recognition features. Five different features are shown as following:

1) Cumulative width

2) Logarithmic cumulative width

3) Cumulative interval boundary value

4) The boundary value

5) Contradiction interval statistics

In order to verify the accuracy of this new fault diagnosis method, the four fault state (normal state, discharge faults, overheated faults and grounding faults) p-boxes are involved in the test.

\section{B. Diagnosis with SVM method}

The commonly used kernel functions of Support vector machines are: linear kernel, polynomial kernel function, Gaussian radial basis function and so on. The test results by using different kernel functions are shown in Table 1.

As shown on Table 1, with the same testing sample data and training data, using different kernel functions will lead to different correct recognition rate. The results of the Gaussian RBF kernel function are best. The Gaussian radial basis is chosen as kernel function. In the classification process, the failure pattern is identified by the identifier of the data.

TABLE 1 COMPARISON OF DIFFERENT KERNEL FUNCTION CLASSIFICATION RESULTS

\begin{tabular}{|c|c|c|c|c|}
\hline \multirow{2}{*}{$\begin{array}{c}\text { the types of } \\
\text { kernel functions }\end{array}$} & \multicolumn{4}{|c|}{ The correct diagnosis rate (\%) } \\
\cline { 2 - 5 } & $\begin{array}{c}\text { normal } \\
\text { state }\end{array}$ & $\begin{array}{c}\text { discharge } \\
\text { fault }\end{array}$ & $\begin{array}{c}\text { overheated } \\
\text { fault }\end{array}$ & $\begin{array}{c}\text { Grounding } \\
\text { fault }\end{array}$ \\
\hline $\begin{array}{c}\text { Linear } \\
\text { kernel }\end{array}$ & 98 & 89 & 91 & 90 \\
\hline $\begin{array}{c}\text { Polynomial } \\
\text { kernel }\end{array}$ & 97 & 85 & 98 & 88 \\
\hline $\begin{array}{c}\text { Gaussian } \\
\text { radial basis }\end{array}$ & 100 & 95 & 92 & 99 \\
\hline
\end{tabular}

The identifier of the normal state, discharge faults, overheated faults and grounding faults is defined as F1, F2, F3, and F4 respectively. The diagnosis results are shown in Table 2.

TABLE 2 THE CLASSIFICATION RESULTS WITH THE GAUSSIAN RBF KERNEL FUNCTION

\begin{tabular}{|c|c|c|c|c|}
\hline \multirow{2}{*}{ Fault pattern } & \multicolumn{4}{|c|}{ The correct recognition rate (\%) } \\
\cline { 2 - 5 } & F1 & F2 & F3 & F4 \\
\hline normal & 100 & 0 & 0 & 0 \\
\hline $\begin{array}{c}\text { discharge } \\
\text { fault }\end{array}$ & 0 & 98 & 3 & 0 \\
\hline $\begin{array}{c}\text { overheated } \\
\text { fault }\end{array}$ & 0 & 5 & 97 & 0 \\
\hline $\begin{array}{c}\text { Grounding } \\
\text { fault }\end{array}$ & 0 & 0 & 0 & 99 \\
\hline
\end{tabular}

As shown on Table 2, the use of multi-class support vector machine for bearing fault diagnosis is fast, not sensitive to noise. The method has high diagnosis rate with high accuracy. Moreover, it is not sensitive to the uncertainty of p-boxes and adapted to some extent overlap among p-boxes, which proves that this identification method has good generalization ability.

\section{CONCLUSIONS}

In order to solve the problem of small sample and improve the diagnosis ability of power transformer by analyzing the dissolved gas, a new method based on probability boxes theory was proposed. To take advantage of the correlation and complementation from the different information sources, the $\mathrm{p}$ boxes had been fused. The SVM method has great advantage in fault diagnosis. Extracting different types of cumulative uncer- tainty measures from p-boxes can establish the SVM Features Database. The analysis result shows that the combination of $\mathrm{p}$ box and SVM can achieve a high diagnosis rate.

\section{ACKNOWLEDGEMENT}

The corresponding author of this paper is Du Yi. The research work was supported by the Applied Fundamental Research Project of Yunnan Province (No. 2013FZ020), the natural science foundation of China (No. 51365020), Personnel Training Project of Yunnan Province (KKSY201303095).

\section{REFERENCES}

[1] Nareh R,Sharma V,Vashisth M. An integrated neural fuzzy approach for fault diagnosis of transformers[J]. IEEE Transactions on Power Delivery,2008,23(4):2017-2024

[2] Ji Hang,Zhu Yongli,Guo Wei.Research of transformer condition grading based on fuzzy syntheis evaluation[J]. Relay,2006,34(5);29-33

[3] Liu Tongjie,Liu Zhigang,Han Zhiwei.Application of adaptive fuzzy support vector machine with adjacent incremental algorithm of transformer fault diagnosis[J]. Power System Protection and Control,2010,38(17):47-52.

[4] Ahmed, M.R.,Geliel, M.A.,Khalil, A. Power transformer fault diagnosis using fuzzy logic technique based on dissolved gas analysis, 2013 21st Mediterranean Conference on Control and Automation, MED 2013 Conference Proceedings, p 584-589, 2013.

[5] Klir GJ: Uncertainty and Information: Foundations of Generalized Information Theory. Wiley-IEEE Press, 2005

[6] Ferson. Constructing Probability Boxes and Dempster-Shafer Structures [M]. Sandia National Laboratories, 2003:143-180

[7] Berleant, Bounding. The Times to Failure of 2-Components Systems. IEEE Transaction on Reliability, 2004, 53(4):542-550

[8] Fulvio Tonon. Using random set theory to propagate epistemic uncertainty through a mechanical system. Reliability Engineering and System Safety, 2004, 85:169-181

[9] Kriegler, Held. Utilizing belief functions for the estimation of future climate change. International Journal of Approximate Reasoning, 2005, 39:185-209

[10] Scott Ferson, Troy Tucker. Sensitivity in risk analyses with uncertain numbers. Applied Biomathematics, Setauket, New York, 2005

[11] Ferson, Tucker. Sensitivity analysis using probability bounding. Reliability engineering and system safety, 2006, 91(10): 1435-1442

[12] Limbourg, Savic. Fault tree analysis in an early design stage using the Dempster-Shafer theory of evidence. Risk, Reliability and Societal Safety. London : Aven \& Vinnem (eds) Taylor \& Francis Group, 2007

[13] Michael Oberguggenberger, Julian King, Bernhard Schmelzer. Imprecise probability methods for sensitivity analysis in engineering. In 5th International Symposium on Imprecise Probability: Theories and Applications, Prague, Czech Republic, 2007, 6: 1130-1138

[14] Vapnik V N. Statistical learning theory [M]. Xu JianHua, Zhang XueGong, trans. Berjing: Publishing House of Electronics Industry, 2006. 